\title{
Structure and Properties of Hydrophobic Aggregation Hydrogel with Chemical Sensitive Switch
}

\author{
Jiufang Duan and Jianxin Jiang \\ MOE Engineering Research Center of Forestry Biomass Materials and Bioenergy, Beijing Forestry University, Beijing 100083, China \\ Correspondence should be addressed to Jiufang Duan; duanjiu99@163.com
}

Received 8 May 2017; Revised 28 July 2017; Accepted 5 September 2017; Published 9 October 2017

Academic Editor: Bernabé L. Rivas

Copyright (C) 2017 Jiufang Duan and Jianxin Jiang. This is an open access article distributed under the Creative Commons Attribution License, which permits unrestricted use, distribution, and reproduction in any medium, provided the original work is properly cited.

\begin{abstract}
Hydrogels with chemical sensitive switch have control release properties in special environments. A series of polyacrylamideoctadecyl methacrylate hydrogels crosslinked by $\mathrm{N}, \mathrm{N}^{\prime}$-bis (acryloyl) cystamine were synthesized as potential chemical sensitive system. When this hydrogel encounters dithiothreitol it can change its quality. The properties of the hydrogels were characterized by infrared spectroscopy, contact angle, and scanning electron microscopy. The water absorption of the hydrogel has the maximum value of $475 \%$, when the content of octadecyl methacrylate is $5 \mathrm{wt} \%$. The amount of weight loss was changed from $34.6 \%$ to $17.2 \%$, as the content of octadecyl methacrylate increased from $3 \mathrm{wt} \%$ to $9.4 \mathrm{wt} \%$. At the same time, the stress of the hydrogel decreased from $67.01 \%$ to $47.61 \%$; the strength of the hydrogel reaches to the maximum $0.367 \mathrm{Mpa}$ at $7 \mathrm{wt} \%$ octadecyl methacrylate. The increasing content of octadecyl methacrylate from $3 \mathrm{wt} \%$ to $9.4 \mathrm{wt} \%$ can enhance the hydrophobicity of the hydrogel; the contact angle of water to hydrogel changed from $14.10^{\circ}$ to $19.62^{\circ}$. This hydrogel has the porous structure which permits loading of oils into the gel matrix. The functionalities of the hydrogel make it have more widely potential applications in chemical sensitive response materials.
\end{abstract}

\section{Introduction}

Hydrogels usually are prepared by hydrophilic polymers with highly swollen properties, as well as crosslinked threedimensional structures. Recently studies have been done on hydrogels with mechanical properties and superporous, selfhealing, self-assembling, and stimuli-sensitive hydrogels [1$3]$. They can be used as wound dressings and in tissue engineering, drug delivery systems, and water treatment agents [4-8].

Stimuli-sensitive hydrogels have become the subject of extensive studies as intelligent materials, because of their multifunctionality in different environments, such as $\mathrm{pH}$, temperature, ultrasound, electric fields, and chemical reagents [9-13]. When these hydrogels are placed in these environments, they change their structure by themselves. Accordingly stimuli-sensitive hydrogels have potential applications in drug carriers by determining the selected solute, removing toxins, recycling, and separating products related to the industrial process.
The problems relating to the smart control of hydrogels were highly concerned [14]. Hydrophobic aggregate is critical to improving the mechanical properties of hydrogels by stress dissipation mechanisms. Aqueous polymer with hydrophobic segments can aggregate at aqueous environment and can be transformed into hydrogels. And disulfide bonds can be designed as a reversible switch in hydrogel design [15]. Therefore, by combining hydrophobic segments with the reversible switch systems, the intelligent hydrogel with sense of chemical changes can be applied in a wide range of applications. As a result, it enables us to make smart systems in separation of specific substances and drug control delivery systems.

Here we reported a chemical sensitive hydrogel and how it responds to dithiothreitol (DTT) in water solution. This material was made by grafting the octadecyl methacrylate and disulfide bond to the PAM. Therefore DTT triggered a quality change owing to breaking the crosslinked S-S bond, and the hydrogel showed the sense of chemical changes properties because of octadecyl methacrylate. The properties of 


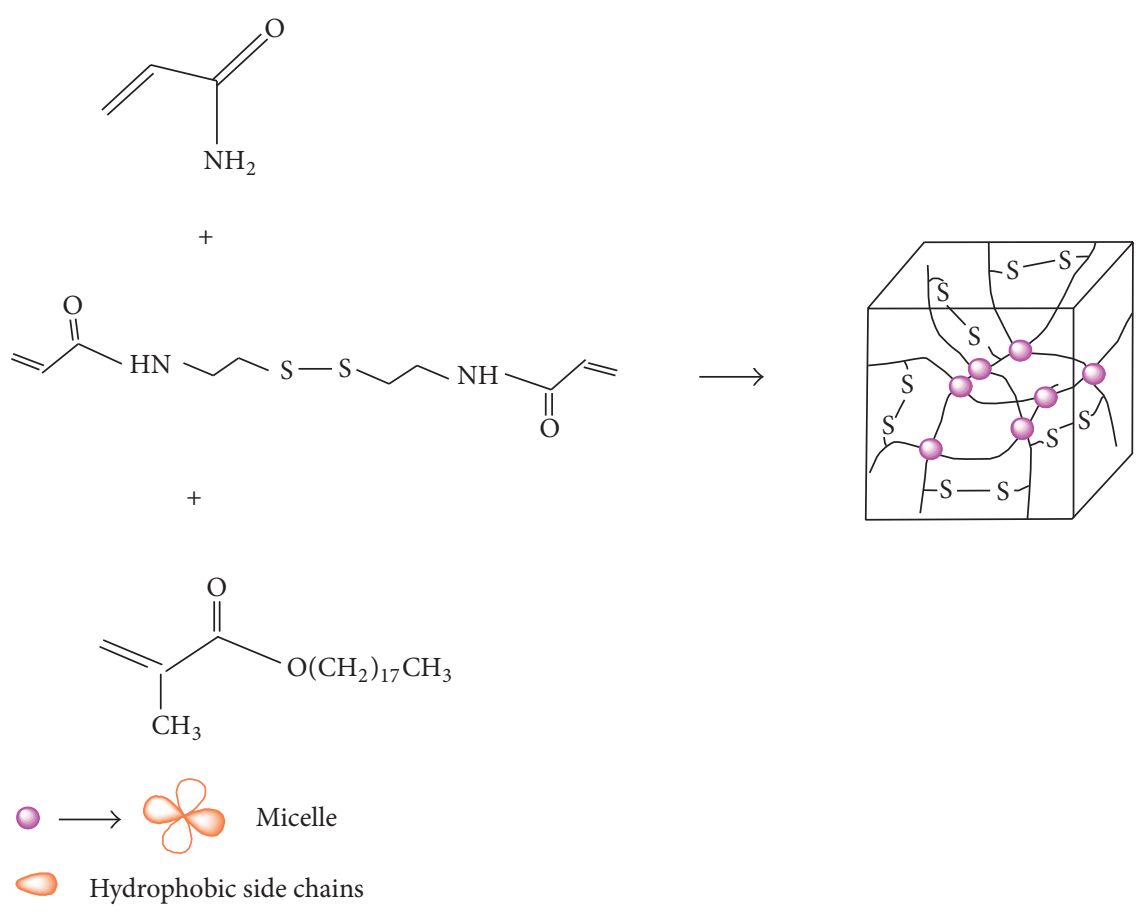

FIGURE 1: The equation of octadecyl methacrylate-acrylamide- $\mathrm{N}, \mathrm{N}^{\prime}$-bis(acryloyl)-cystamine hydrogel.

the hydrogel were characterized by mechanic strength and contact angle of water measurements.

\section{Experimental Work}

2.1. Materials and Methods. Acrylamide, stearyl methacrylate, ammonium persulfate (APS), sodium dodecyl sulfate (SDS), N, $\mathrm{N}^{\prime}$-bis (acryloyl) cystamine, $\mathrm{N}, \mathrm{N}^{\prime}, \mathrm{N}, \mathrm{N}^{\prime}$-tetramethylethylenediamine (TEMED), and $\mathrm{NaCl}$ were commercially available and used as received.

IR spectra were recorded by FTIR (Nicolet iN10 Thermo Fisher Scientific China) over the region from 4000 to $400 \mathrm{~cm}^{-1}$. For morphological characterization, the hydrogels were analysed by scanning electron microscope (SEM) (S-3400N, Hitachi, Japan) with an acceleration voltage of $40 \mathrm{kV}$. A thin layer of the sample was cast on a silica wafer and freeze-dried, overnight, in a lyophiliser. A layer of gold was sputter-coated over the sample by vacuum spray to forma conductive surface.

Tensile stress-strain measurements were performed by using an Instron 3365 Universal Testing Machine (Norwood, MA, USA) with the following parameters: sampling rate: $10.000 \mathrm{pts} / \mathrm{sec}$; beam speed: $100 \mathrm{~mm} / \mathrm{min}$; full scale load range: $0.1000 \mathrm{kN}$; humidity: $25 \%$; and temperature: $24^{\circ} \mathrm{C}$.

The cylindrical shaped gel samples measured $60 \mathrm{~mm} \times$ $10 \mathrm{~mm}$. Each data point was measured on six samples, and the average value of five measurements was taken. Statistical analysis of data was performed by one-way analysis of variance, assuming a confidence level of $95 \%(P<0.05)$ for statistical significance.

The gravimetric method was used to measure the swelling ratios of the gels. After immersion in distilled water for approximately $48 \mathrm{hr}$ at $25^{\circ} \mathrm{C}$ to reach swelling equilibrium, the gel samples were weighed. The average value of three measurements was taken. The equilibrium swelling ratio (SR) was calculated as $\mathrm{SR}=W_{s} / W_{d}$, where $W_{s}$ is the weight of the swollen gel and $W_{d}$ is the weight of the gel in its dry state.

2.2. Synthesis of the Octadecyl Methacrylate-acrylamide- $N, N^{\prime}$ bis(acryloyl)-cystamine Hydrogel. The gels were synthesized by micellar copolymerization. The reaction system was generally started by dissolving $2.065 \mathrm{~g} \mathrm{NaCl}$ and $4.95 \mathrm{~g}$ sodium dodecyl sulfate into $70 \mathrm{~mL} 25 \%$ ethanol at $60^{\circ} \mathrm{C}$ to obtain a transparent solution. Then, $6.475 \mathrm{~g}$ acrylamide, $0.719 \mathrm{~g} \mathrm{~N}, \mathrm{~N}^{\prime}-$ bis (acryloyl) cystamine, and stearylmethacrylate (3 wt.\%, 4 wt.\%, 5 wt.\%, 7 wt.\%, and $9.4 \%$ relative to the amount of solid content) were dissolved in this solution under stirring for $2 \mathrm{~h}$ at a temperature of $35^{\circ} \mathrm{C} .175 \mu \mathrm{L}$ TEMED was added to the solution hereafter. Finally, $0.08 \mathrm{~g}$ of APS was added to initiate the reaction. The copolymerization reactions were carried out at $25^{\circ} \mathrm{C}$ for $24 \mathrm{~h}$.

\section{Results and Discussion}

3.1. Synthesis of Hydrogels. Acrylamide (AM), octadecyl methacrylate, and $N, N^{\prime}$-bis(acryloyl)-cystamine form an amphipathic copolymer at this system (Figure 1). C18 acts as hydrophobic parts and AM act as hydrophilic parts, and $\mathrm{N}, \mathrm{N}^{\prime}$-bis(acryloyl)-cystamine acts as the cross-linking agent, which connects the polymer into a three-dimensional network structure. The hydrophobic parts can also form the micelles in the hydrogel, which can be used to hold hydrophobic particles in hydrophilic environments. 


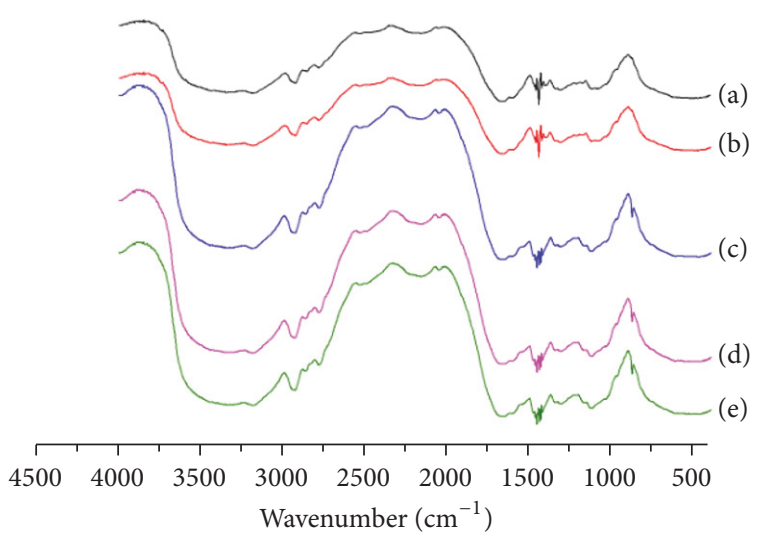

FIGURE 2: FTIR spectra of octadecyl methacrylate-acrylamide$\mathrm{N}, \mathrm{N}^{\prime}$-bis(acryloyl)-cystamine hydrogel (concentration of octadecyl methacryl (a) 3\%, (b) 4\%, (c) 5\%, (d) 7\%, and (e) $9 \%$ ).

The dithiothreitol cause the disulfide bond to break down into -SH groups [15]. Correspondingly, the disappearance of the connecting bonds will lead to its structure changing from a three-dimensional network to a soluble linear structure. At the same time the hydrogel changes the quality. Disulfide bond (-S-S-) is a smart covalent bond, it can broken down into - $\mathrm{SH}$ groups when it encounters the dithiothreitol [15]. Accompanied by breakdown of the disulfide bonds in the gel, the hydrogel changes its quality.

3.2. Characterization of the Hydrogels. The FTIR spectra of AM, octadecyl methacrylate, and $\mathrm{N}, \mathrm{N}^{\prime}$-bis(acryloyl)-cystamine crosslinked hydrogels are presented in Figure 2. The prominent band at $2929 \mathrm{~cm}^{-1}$ was attributed to the asymmetric absorption peak of methylene- $\mathrm{CH}_{2}$. The bands at $1500 \mathrm{~cm}^{-1}$ and $918 \mathrm{~cm}^{-1}$ correspond to the bending vibration of $\mathrm{N}-\mathrm{H}$ and the stretching vibration of $\mathrm{C}-\mathrm{N}$, respectively. The peak at $1446 \mathrm{~cm}^{-1}$ corresponds to the bending vibration absorption peak of $\mathrm{C}-\mathrm{H}$ bonds. According to Figure 2, the intensity of the stretching vibration of methylene increased with the content of the octadecyl methacrylate.

3.3. The Equilibrium Swelling Ratio of the Octadecyl Methacrylate-acrylamide- $N, N^{\prime}$-bis(acryloyl)-cystamine Hydrogels. Hydrogels of octadecyl methacrylate-acrylamide-N, $\mathrm{N}^{\prime}$ bis(acryloyl)-cystamine could absorb large amounts of water. The equilibrium swelling ratio of the hydrogel reached $95 \%$ at $1 \mathrm{~h}$ and formed a saturation state after immersion in water for $6 \mathrm{~h}$. When the concentration of the hydrophobic parts (octadecyl methacrylate) was changed from $3 \%$ to $9 \%$, the equilibrium swelling ratio could reach the highest value of $475 \%$ at $5 \%$ percent of octadecyl methacrylate (Figure 3 ). The possible reason is that the increase of octadecyl methacrylate can create more hydrophobic micelles, which give more cross-linking points in the hydrogel. However, too many hydrophobic cross-linking points will affect the spread of water, so the water absorption ability of hydrogels was decreased.

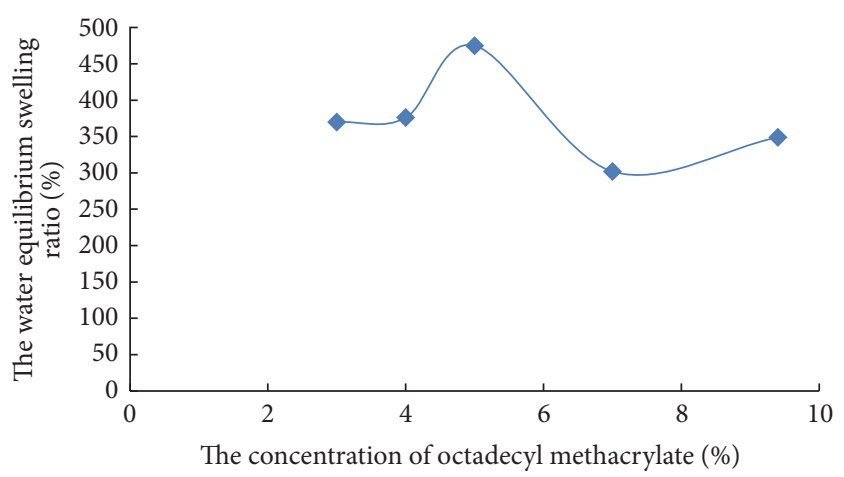

FIGURE 3: The solution absorption ratio of octadecyl methacrylateacrylamide-N, $\mathrm{N}^{\prime}$-bis(acryloyl)-cystamine hydrogel.

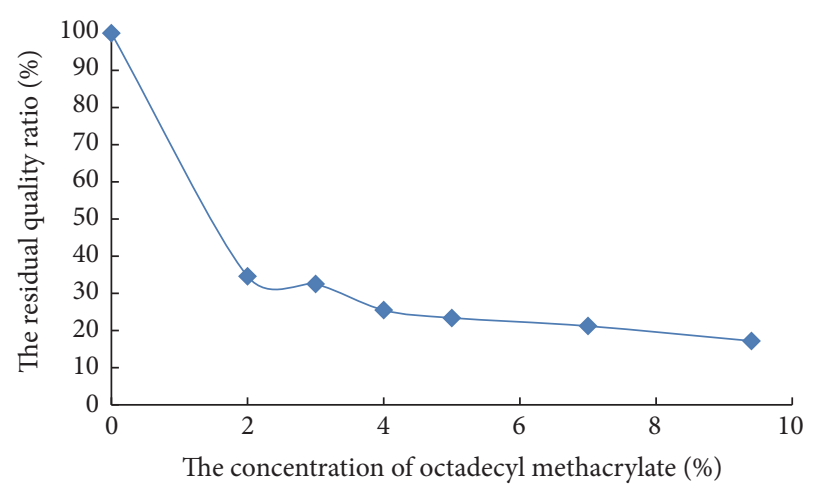

FIgure 4: The residual quality ratio of octadecyl methacrylateacrylamide- $\mathrm{N}, \mathrm{N}^{\prime}$-bis(acryloyl)-cystamine hydrogel (the concentration of octadecyl methacrylate (a) $3 \%$, (b) $4 \%$, (c) $5 \%$, (d) $7 \%$, and (e) $9 \%)$.

3.4. The Residual Quality Ratio of Octadecyl Methacrylateacrylamide- $N, N^{\prime}$-bis(acryloyl)-cystamine Hydrogel. When the concentration of octadecyl methacrylate changed from $3 \%$ to $9 \%$, the sol ratio decreased from $34.6 \%$ to $17.2 \%$, as shown at Figure 4. This was caused by the increase of hydrophobic micelles caused by more octadecyl methacrylate. More hydrophobic micelles will induce more cross-linking points in the molecular chain, which make the molecular chain retain a cross-linking state after the reduction of disulfide reduction. Correspondingly, the residual quality ratio was decreased.

3.5. Mechanical Properties of the Octadecyl Methacrylate-acrylamide-N, $N^{\prime}$-bis(acryloyl)-cystamine Hydrogel. The hydrophobic parts octadecyl methacrylate can affect the mechanical properties of the octadecyl methacrylate-acrylamide-N, $\mathrm{N}^{\prime}$ bis(acryloyl)-cystamine hydrogel. The highest compressive strength $137.8 \mathrm{KPa}$ of the hydrogel can reach $5 \%$ of octadecyl methacrylate (Figure 5). The strain of the hydrogel was increased as the octadecyl methacrylate concentration changed from $2 \%$ to $5 \%$, while a sharp decline occurred while the octadecyl methacrylate concentration changed from $5 \%$ to $9 \%$ (Figure 6). More octadecyl methacrylate can increase the strain and compressive strength of the hydrogel 


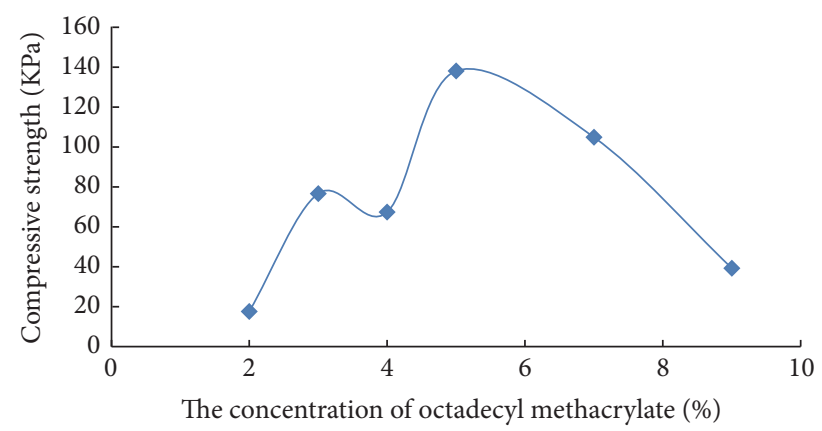

FIGURE 5: The mechanical strength of the octadecyl methacrylateacrylamide- $\mathrm{N}, \mathrm{N}^{\prime}$-bis(acryloyl)-cystamine hydrogel.

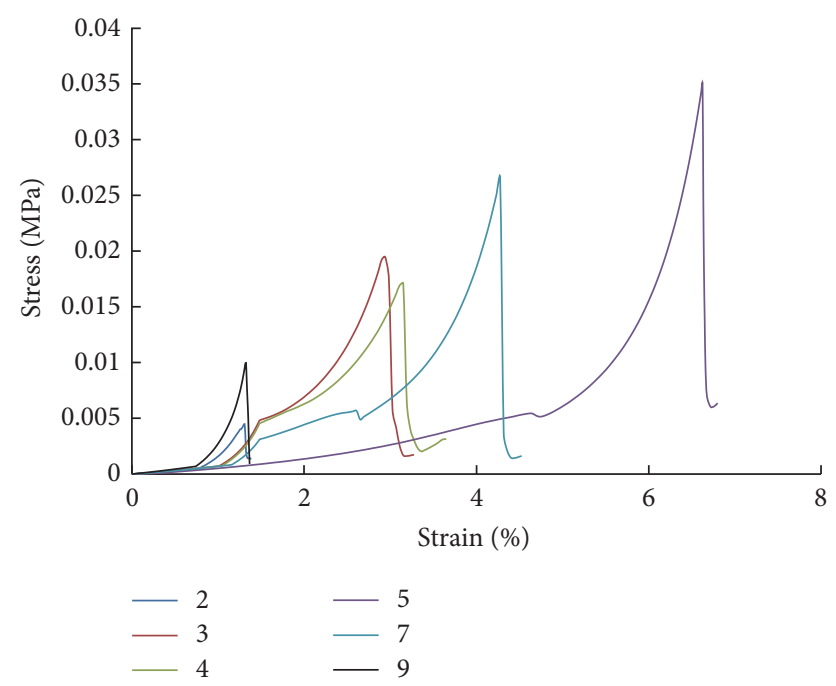

FIGURE 6: The compressive stress/strain capacity curves of octadecyl methacrylate-acrylamide- N, $\mathrm{N}^{\prime}$-bis(acryloyl)-cystamine (octadecyl methacrylate concentration: $2 \%, 3 \%, 4 \%, 5 \%, 7 \%$, and $9 \%$ ).

by forming more cross-linking points. However, too many cross-linking points will decrease the strain and compressive strength [16].

3.6. The Contact Angle with Water. The contact angle with water of the material can reflect the hydrophobic properties of this material. The contact angle of the hydrogel increased from $14.10^{\circ}$ to $19.62^{\circ}$, when the concentration of octadecyl methacrylate was changed at the range of $3 \%$ to $9.4 \%$ (Figure 7). This result shows that the long alkane structure of octadecyl methacrylate can increase the hydrophobic ability of the hydrogel.

3.7. Surface Morphology of Octadecyl Methacrylate-acrylamide- $N, N^{\prime}$-bis(acryloyl)-cystamine Hydrogel. Figure 8 shows SEM micrographs of freeze-dried octadecyl methacrylateacrylamide-N, $\mathrm{N}^{\prime}$-bis(acryloyl)-cystamine hydrogel. This hydrogel has a highly porous structure. The porosity property permits loading of water and hydrophobic particles into the gel matrix.

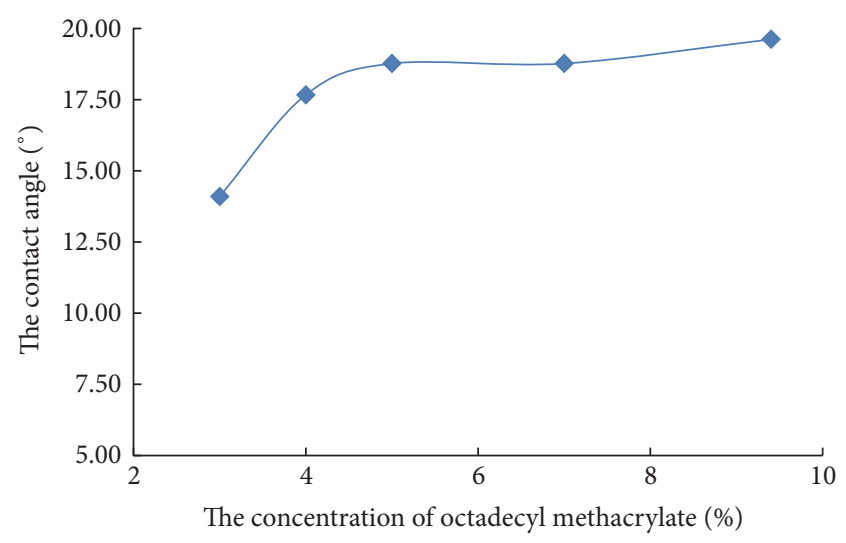

FIGURE 7: The contact angle of octadecyl methacrylate-acrylamide$\mathrm{N}, \mathrm{N}^{\prime}$-bis(acryloyl)-cystamine hydrogel.

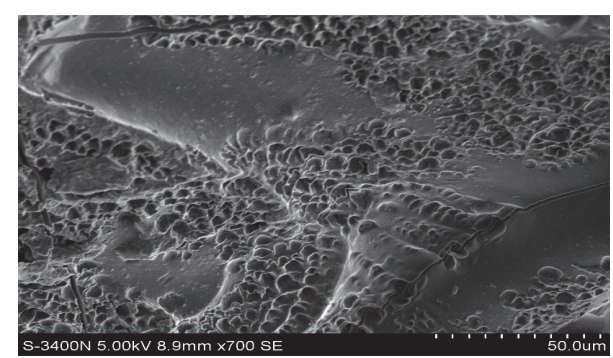

FIGURE 8: The SEM image of octadecyl methacrylate-acrylamide$\mathrm{N}, \mathrm{N}^{\prime}$-bis(acryloyl)-cystamine hydrogel.

\section{Conclusions}

In this study, octadecyl methacrylate-acrylamide- $\mathrm{N}^{\prime} \mathrm{N}^{\prime}$ bis(acryloyl)-cystamine hydrogel was prepared in an aqueous solution environment. The hydrogel exhibited swelling changes, mechanical strength changes, hydrophobic ability changes, and ratio of degradation changes in response to octadecyl methacrylate concentration. The hydrogel is also a kind of stimuli-responsive hydrogel that undergoes abrupt changes in quality in response to dithiothreitol. This has potential applications in biomedicine or other systems that need "intelligent" responses.

\section{Conflicts of Interest}

The authors declare that there are no conflicts of interest regarding the publication of this paper.

\section{Acknowledgments}

This research was financially supported by the China Ministry of Science and Technology (2016YFD0600803) and the National Natural Science Foundation of China (31600464).

\section{References}

[1] C. H. Cho, S. Kwon, and J. Park, "Assembly of hydrogel units for 3D microenvironment in a poly(dimethylsiloxane) channel," Micro and Nano Systems Letters, vol. 5, no. 1, 2017. 
[2] Y. Qiu and K. Park, "Environment-sensitive hydrogels for drug delivery," Advanced Drug Delivery Reviews, vol. 53, no. 3, pp. 321-339, 2001.

[3] A. Phadke, C. Zhang, B. Arman et al., "Rapid self-healing hydrogels," Proceedings of the National Academy of Sciences of the United States of America, vol. 109, no. 12, pp. 4383-4388, 2012.

[4] S. W. Benner and C. K. Hall, "Nanoparticle-induced assembly of hydrophobically modified chitosan," Molecular Simulation, vol. 43, no. 9, pp. 664-674, 2017.

[5] S. Peng, C.-W. Wu, J.-Y. Lin, C.-Y. Yang, M.-H. Cheng, and I.-M. $\mathrm{Chu}$, "Promoting chondrocyte cell clustering through tuning of a poly(ethylene glycol)-poly(peptide) thermosensitive hydrogel with distinctive microarchitecture," Materials Science and Engineering $C$, vol. 76, pp. 181-189, 2017.

[6] S. J. Boardman, R. Lad, D. C. Green, and P. D. Thornton, "Chitosan hydrogels for targeted dye and protein adsorption," Journal of Applied Polymer Science, vol. 134, no. 21, Article ID 44846, 2017.

[7] A. Kumar, K. M. Rao, and S. S. Han, "Synthesis of mechanically stiff and bioactive hybrid hydrogels for bone tissue engineering applications," Chemical Engineering Journal, vol. 317, pp. 119-131, 2017.

[8] B. Singh, S. Sharma, and A. Dhiman, "Acacia gum polysaccharide based hydrogel wound dressings: Synthesis, characterization, drug delivery and biomedical properties," Carbohydrate Polymers, vol. 165, pp. 294-303, 2017.

[9] L. Wang, Y. Long, H. Ding, J. Geng, and B. Bai, “Mechanically robust re-crosslinkable polymeric hydrogels for water management of void space conduits containing reservoirs," Chemical Engineering Journal, vol. 317, pp. 952-960, 2017.

[10] A. Osman, E. T. Oner, and M. S. Eroglu, "Novel levan and pNIPA temperature sensitive hydrogels for 5-ASA controlled release," Carbohydrate Polymers, vol. 165, pp. 61-70, 2017.

[11] H. Jiang and T. Kobayashi, "Ultrasound stimulated release of gallic acid from chitin hydrogel matrix," Materials Science and Engineering C, vol. 75, pp. 478-486, 2017.

[12] P. Treenate and P. Monvisade, "In vitro drug release profiles of $\mathrm{pH}$-sensitive hydroxyethylacryl chitosan/sodium alginate hydrogels using paracetamol as a soluble model drug," International Journal of Biological Macromolecules, vol. 99, pp. 71-78, 2017.

[13] K. Depa, A. Strachota, M. Šlouf, J. Brus, and V. Cimrová, "Synthesis of conductive doubly filled poly(N-isopropylacrylamide)-polyaniline-SiO2 hydrogels," Sensors and Actuators, B: Chemical, vol. 244, pp. 616-634, 2017.

[14] J. Ge, L. A. Shi, Y. C. Wan et al., "Joule-heated graphenewrapped sponge enables fast clean-up of viscous crude-oil spill," Nature Nanotechnology, vol. 33, no. 2017, pp. 1-8, 2017.

[15] H. A. Aliyar, P. D. Hamilton, and N. Ravi, "Refilling of ocular lens capsule with copolymeric hydrogel containing reversible disulfide," Biomacromolecules, vol. 6, no. 1, pp. 204-211, 2005.

[16] J. Duan, J. Jiang, J. Li, L. Liu, Y. Li, and C. Guan, “The preparation of a highly stretchable cellulose nanowhisker nanocomposite hydrogel," Journal of Nanomaterials, vol. 2015, Article ID 963436, 8 pages, 2015. 

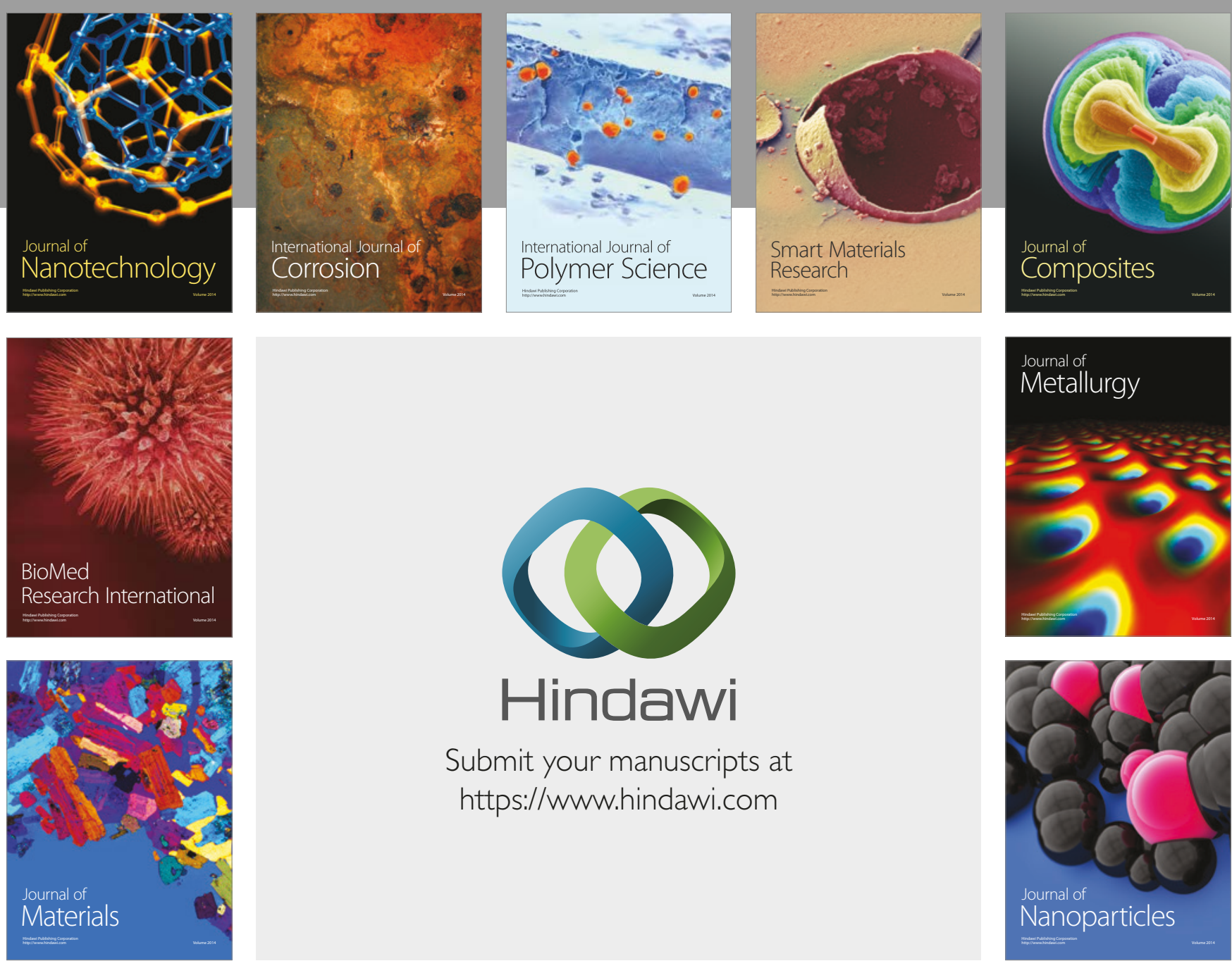

\section{Hindawi}

Submit your manuscripts at

https://www.hindawi.com
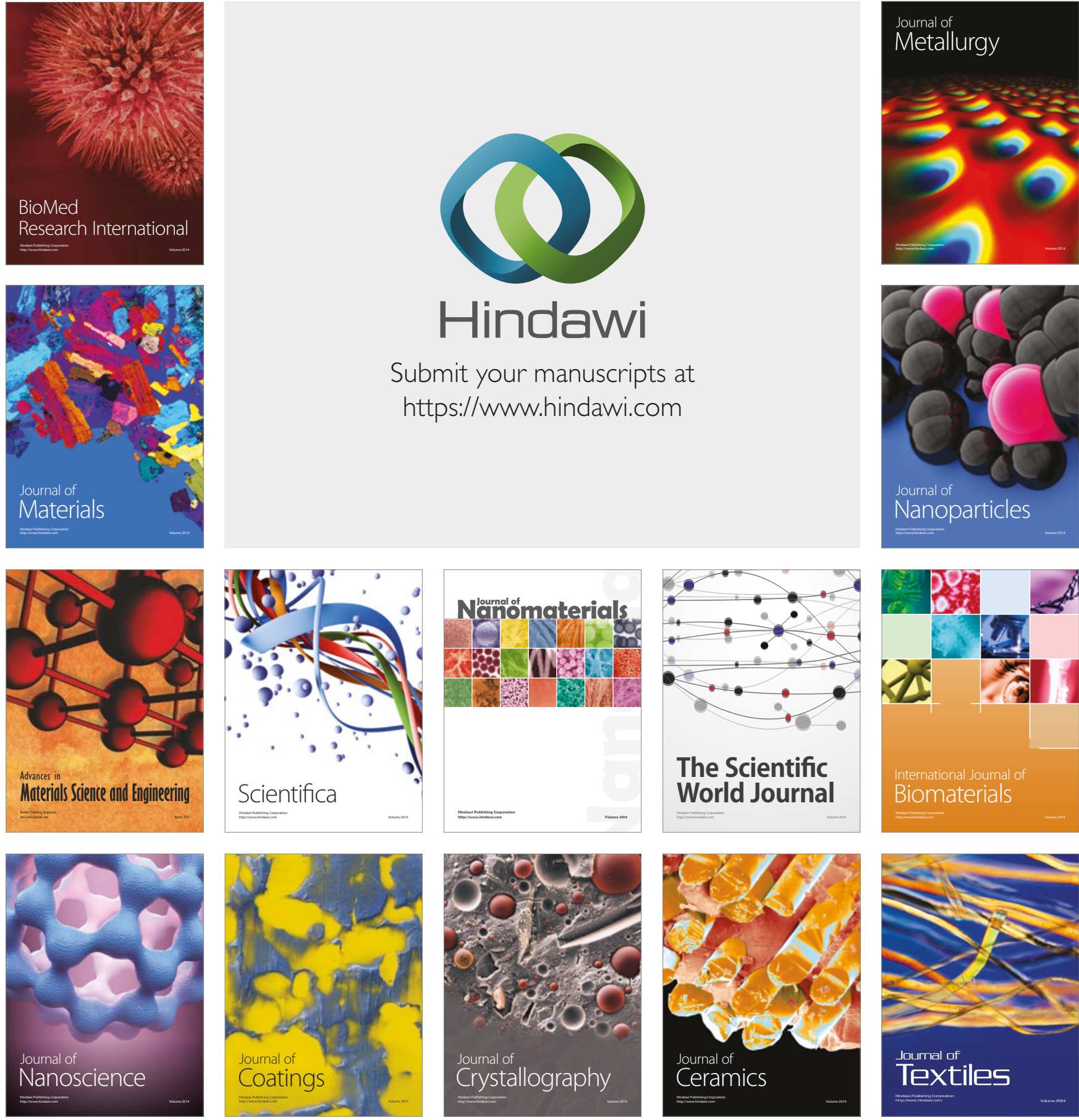

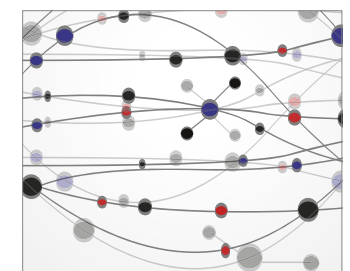

The Scientific World Journal
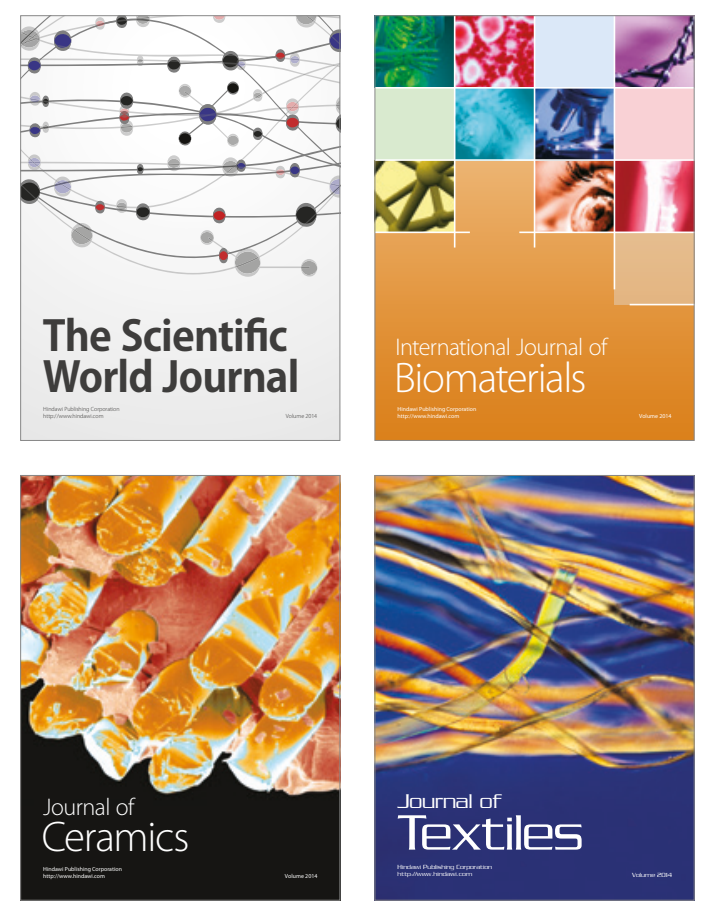